\title{
Déficit de cobre en el lactante
}

Drs. Fubén Puentes R. ${ }^{1,2}$ Ricardo Uauy D. ${ }^{1,3}$ y Carlos Castillo ${ }^{2,3}$

\section{Copper deficit in infants}

\begin{abstract}
Four casts of clinical copper deficiency identified in the course of marasmic protein energy malnutrition are presented.

Age of diagnosis ranged from 4 to 10 months. Alt patients bad one or more bactors associated to copper deficit. Clinical signs included anemia, neutropenia and failure ta thrive in spite of adequate energy and protein intrke. A]] had plasulta copper levels under $50 \mu \mathrm{g} / \mathrm{dl}$. A dramatic response to copper supplementition (ko) $\mu g$-kg-day) was noted in all cases.
\end{abstract}

En el curso del tratamiento recuperacional de lactantes con desnutrición marásmica nos ha correspondido observar diversos casos en que se ha precisado el diagnóstico de déficit de cobre, a través de determinaciones de cenuloplasmina y/o cupremia.

Estos niños han estado hospitalizados en el Servicio de Pediatria y luego algunos han continuado su tratamiento en el Centro de Recuperación Nutricional Macul, dependiente de CONLN.

Hemos seleccionado para esta presentación 4 casos en que el déficit ha sido severo, a juzgar por los valores de laboratorio, con el propósito de resaltar las condiciones en que se puede plantear esta patología en el lactante, así como también revisar su sintomatologia clínica. De lo anterior podrá desprenderse un tratamiento oportuno de esta deficiencia y, en lo posible, su prevención.

\section{CASOS CLINICOS}

\section{A. $E M O(270906)$}

Nace el 2-5-80, por un parto normal, de término, en su domicilio, con un peso de $3.700 \mathrm{~g}$. El embarazo fue también normal. Destaca una pésima situación económico social. Padre alcohólico, sin trabajo, de 40 años. Madre de 29 años, analfabeta, con 7 hijos vivos y uno fallecido (Síndrome de Down y Cardiopatía congénita). Varios hijos son

\footnotetext{
I Departamento de Pediatría Pontilicia Universidad Católióa de Chile.

2Servicio de Pediatría Hospital Dr. Sótero del Aío. Servicio de Salud Metropolitano Sur Oriente.

${ }^{3}$ Instituto de Nutrición y Tecrología de los Alimentus. Liniversidad de Chile.
}

desnutridos. Pésima vivienda, con gran hacinamiento ( 3 personas por cama).

Es hospitalizada por $\mathbf{1}^{\text {a }}$ vez a los 2 meses de edad, con diarrea aguda y deshidratación (enterocolitis) y observación de septicemia bacteriana. Recibía pecho escasamente y Nan. Responde bien al tratamiento antibiótico, hidratación y realimentación. Al egreso, después de 11 días de hospitalización y a pesar de haber subido de peso, está ya con DCP de II grado, de evidente etiología primaria.

Se rehospitaliza a los 6 meses 18 días, por un cuadro de diarrea con deshidratación hipotónica, que evoluciona tórpidamente. Ahora tiene DCP III grado. Se constata intolerancia secundaria a disacáridos y una otitis aguda. Solucionado el problema diarreico, lıego de uso de fómula libre de lactosa y de antibacterianos, se traslada a los 7 meses al Centro Nutricional Macul. Exámenes practicados en ese periodo son normales (proteinemia, hemograma, parasitólogicos de deposiciones, urocultivo, citoquímico y cultivo LCR, sedimento urinario, hemo-cultivos).

Al ingreso a CONIN, se constata cupremia de 14 $\mu \mathrm{g} \%$ (cupremia normal $>90 \mu \mathrm{g} / \mathrm{d}$ ), iniciándose de inmediato tratamiento con sulfato de cobre, subiendo valores a 34 y 91 y $102 \mu \mathrm{g} \%$ a los: $30-60$ y 90 dias de tratamiento.

A pesar de patología intercurrente que presenta en el Centro (Bronquitis a repetición - Varicela impetiginizada, infección enteral por E. Coli enteropatógeno) tiene buena evolución, egresando al año de edad con peso en promedio normal y peso para talla normal.

No se constatô anemia en ningún momento. Hubo neutropenia de 900 granulocitos $\mathrm{x} \mathrm{m}^{3}$ a los 10 meses de edad, que pasa rápidamente y que fue concomitante con un cuadro de varicela. 
B. L.L.A. (261921)

Este menor nace por cesárea el 7-III de 1980, a raíz de ser su madre cesarizada anterior. Pesa al nacer $3.290 \mathrm{~g}$, tiene una talla de $50 \mathrm{~cm}$. y Apgar de VIII al minuto.

El embarazo (2..$^{\circ}$ fue patologico, con toxemia e hidroamnios. Las condiciones económico-sociales de la familia son precarias, con bajo ingreso familiar, padres jóvenes, con escasa o nula educación y mala vivienda.

Ingresa al hospital a los 41 días de vida, constatándose que no había recibido pecho materno, y que la fórmula láctea que se le administraba era hipocalórica e hiperproteica. Se diagnosticó dia-

\section{Gráfico 1}

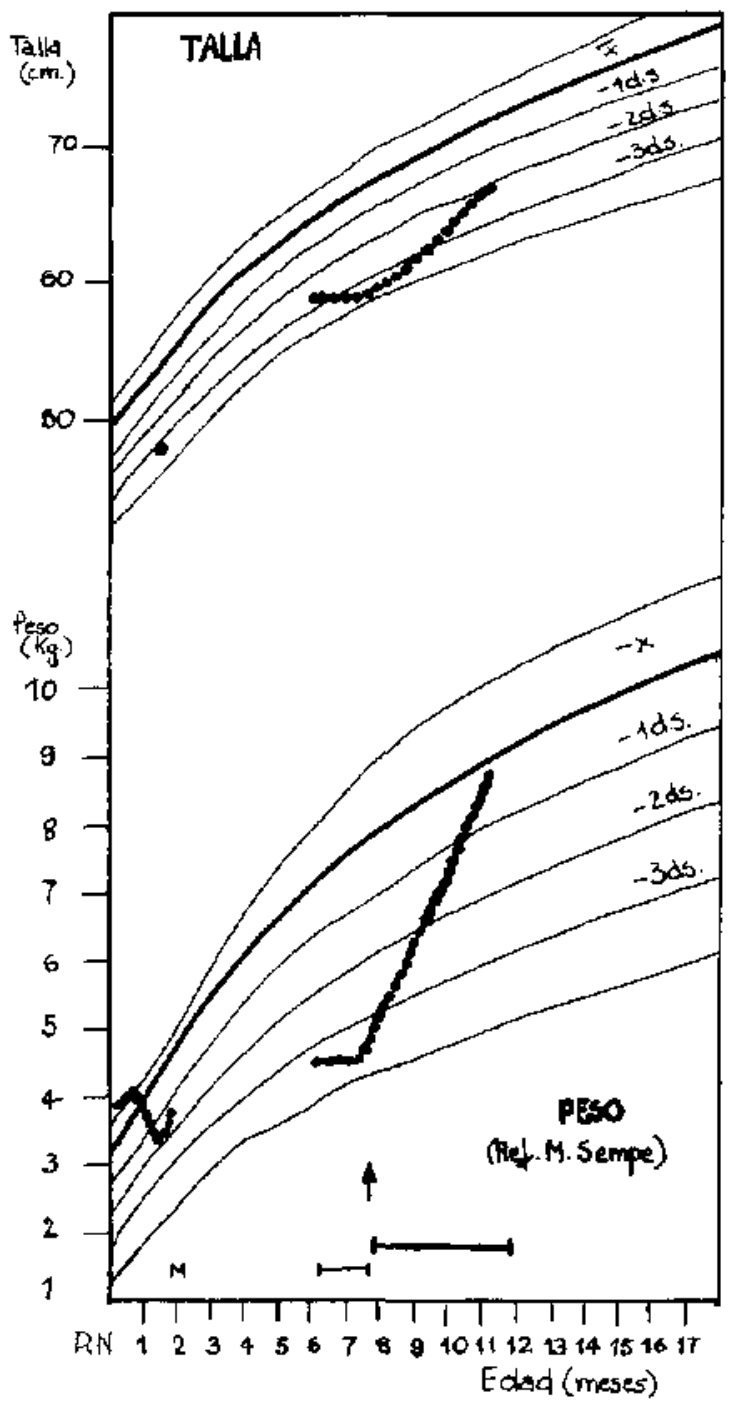

rrea aguda con deshidratación hipertónica. El peso de ingreso fue de $3.020 \mathrm{~g}$. y el de egreso 8 dias después $3.240 \mathrm{~g}$. (DCP I).

Poco después de su alta, llega nuevamente con diarrea aguda y deshidratación al Hospital, constatándose infección enteral bacteriana. Exámenes son normales, entre ellos: hemograma, proteinemia, uroculbivo. Radiografia de huesos largos no muestra tampoco alteraciones. A los 2 meses 28 días se traslada a CONIN, con un peso de $3.750 \mathrm{~g}$. (DCP III G.).

Cupremia tomada en ese momento es normal $(98 \mu \mathrm{g} \%)$. Durante su estada en CONIN presenta una otitis supurada izquierda y una bronconeumonía que requieren tratamiento antibiótico.

A los 6 meses de vida, se diagnostica déficit de cobre por cupremia de $4 \mu \mathrm{g} \%$. Importa destacar que hasta esa edad el nin̄o no había recibido sopa, estando sólo con alimentación láctea artificial, la que fue, además, en algunos periodos insuficiente.

Se agrega sulfato de cobre al tratamiento, con muy buena respuesta, subiendo cupremia a 101 $\mu \mathrm{g} \% 45$ dias después. Al alta (7 1/2 meses) la evaluación del desarrollo psicomotor muestra retraso severo, más acentuado en el área motora.

Posteriormente se controla en forma ambulatoria, continuando su progreso ponderal, llegando al año a un peso de $10 \mathrm{Kg}$. y una talla de $69.5 \mathrm{~cm}$. El desarrollo psicomotor se normaliza en esa edad.

\section{R.M.P. (277543)}

Nace el 22 de junio de 1980 , por parto vaginal. Pesa al nacer $2.100 \mathrm{~g}$. (RN PTAEG) y no tiene problemas en el período neonatal.

Es producto de un primer embarazo, aparentemente normal, en una madre de 17 años, de procedencia rural. Viven varias familias en casa de adobe, en pésimas condiciones. El padre tiene 18 años, con escasos ingresos.

No recibe pecho. A los 3 meses ingresa al hospital por una meningitis purulenta, cuyo diagnóstico etiológico no se logra precisar. Se trata según esquemas habituales con buena respuesta. Alta a los 3 meses 21 días con $4.000 \mathrm{~g}$ de peso.

Reingresa a los 4 meses por una infección urinaria (Echerichia Coli) que también mejora con tratamiento antibacteriano (Cotrimoxasol). Hemograma en esa oportunidad, no muestra anemia ni neutropenia.

Dado que está con DCP III g. se traslada a CON1X, pesquisándose hipocupremia de $47 \mathrm{vg} \%$ 


\section{HOHBRES}

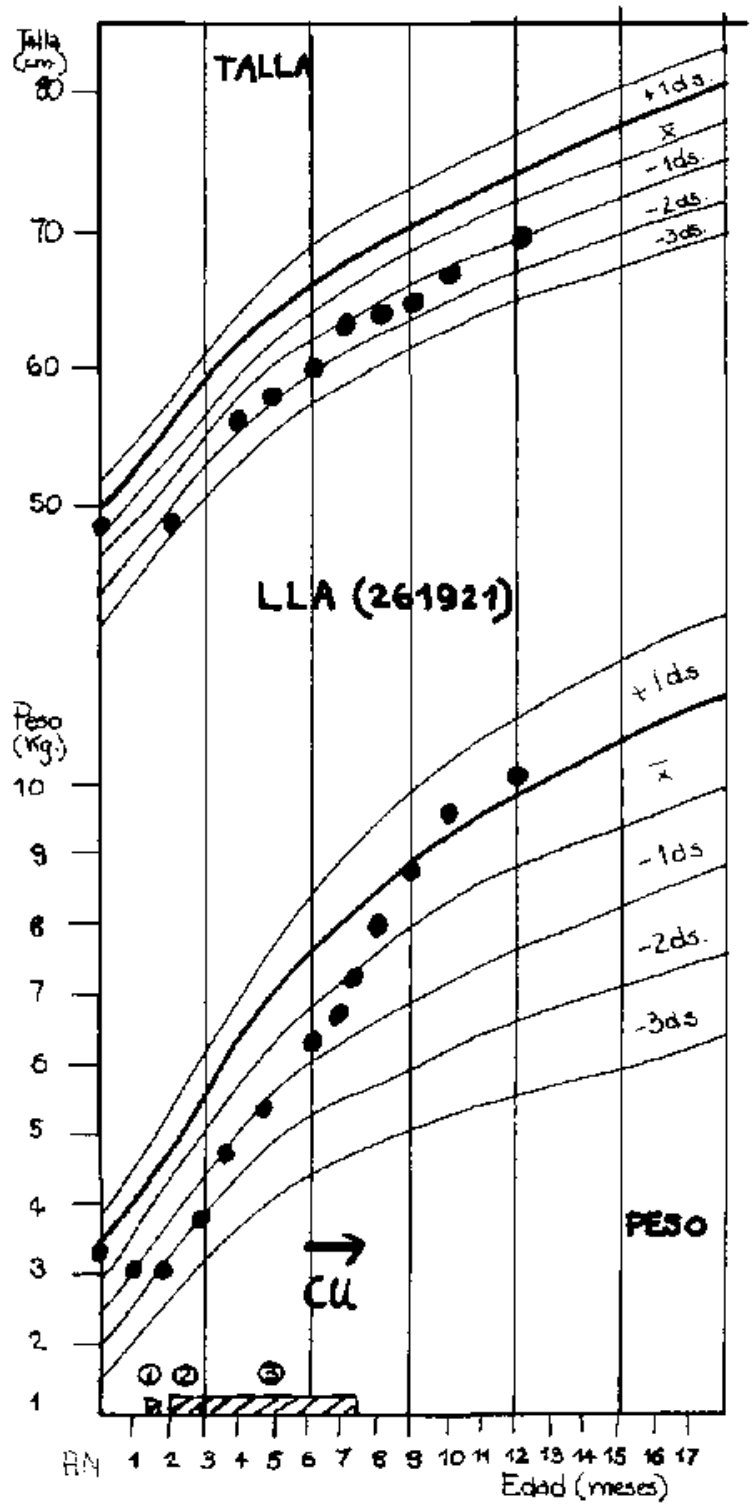

a su ingreso, a los 4 meses de vida. Con adición de sulfato de cobre al tratamiento tiene excelente respuesta ponderal.

Egresa a los 6 meses 22 días con DGP I compensada. Cupremia a los 7 meses 8 días, es normal (118 $\mu \mathrm{g} \%$ ). Controles posteriores ambulatorios permiten apreciar que el menor llega a peso/edad normal.

\section{M.P.S. $(247482)$}

La familia de esta menor presenta, también, pésimas condiciones. La madre de 38 años de

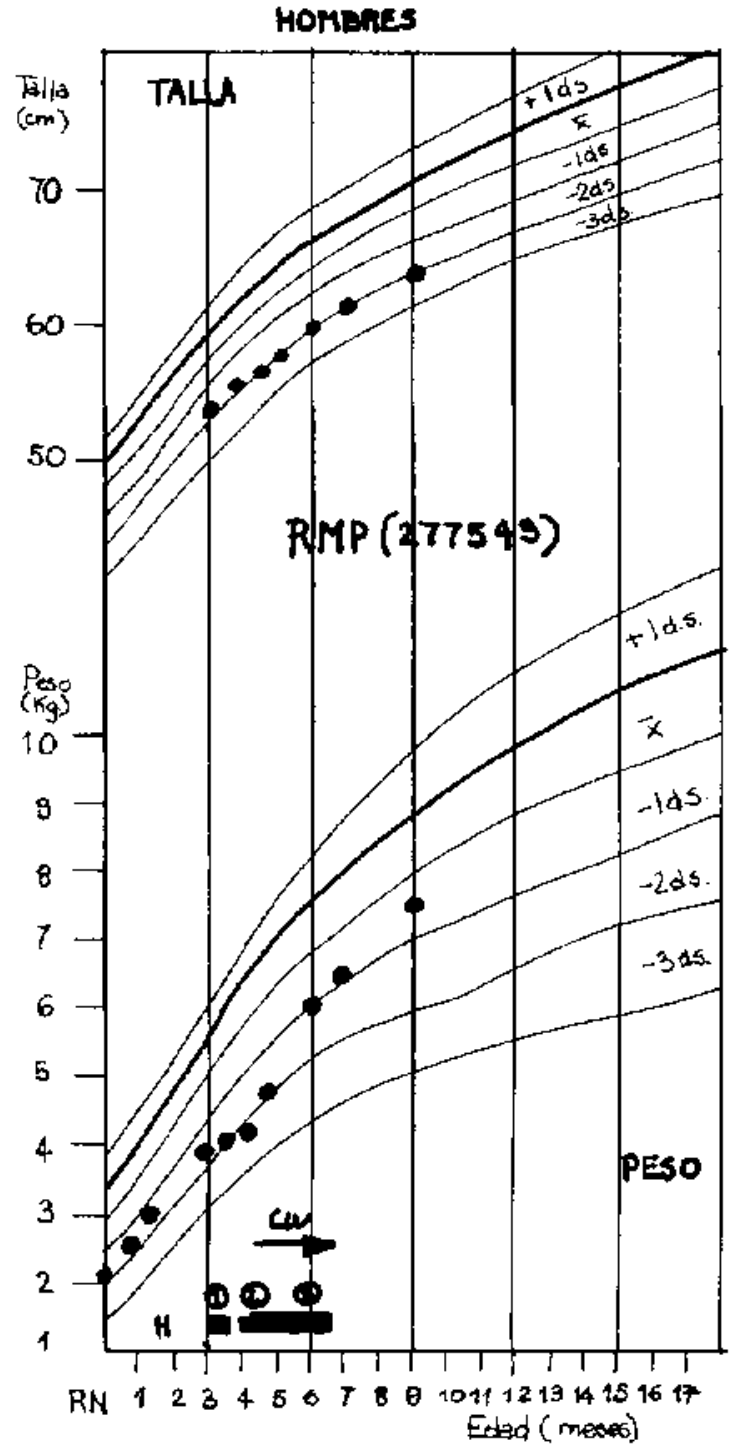

edad tiene 8 hijos vivos, varios de ellos desnutridos, $4 .^{\circ}$ año básico y trabaja en el PEM.

El parto $y$ embarazo fueron nomnales. Nace el l-VI-79 con $3.250 \mathrm{~g}$. de peso y $47 \mathrm{~cm}$. de talla; Apgar VIII al minuto.

No recibió pecho, siendo reemplazado por fórmulas en base a NAN y luego leche Purita.

A los 2 meses 25 días se hospitaliza por primera vez, estando eutrófica, por un cuadro bronconeumónico.

Posteriormente, se vuelve a hospitalizar a los 5 meses y 3 semanas nuevamente por bronconeumonía. Hubo buena respuesta, pero luego presenta cuadro similar, intrahospitalario. Egresa a los 7 
meses, estando ya su estado nutritivo deteriorado (DCP IIg). Exámenes habituales, incluyendo hemograma, son nomales.

Presenta, ambulatoriamente, una $4^{4}$. bronconeumonia. Ingresa a CONIN, a los 7 meses 23 días con DCP III g.

En ese Centro se evidencian claramente 2 etapas, en cuanto a su evolución antropométrica y clínica. La lera.; de aproximadamente 2 meses, se caracteriza por infecciones recurrentes (BNM, Varicela) y curva de peso estacionaria $(-2$ $\mathrm{gm} / \mathrm{Kg} / \mathrm{dia}$ ). La segunda, luego de diagnósticarse hipocupremia e iniciar su tratamiento, evidencia excelente progreso pendoestatural $(9.7 \mathrm{mg} / \mathrm{Kg} /$ día.).

El diagnóstico de déficit de cobre se pesquisó a los 10 1/2 meses luego que, a raíz de proceso agudo respiratorio, se toma hemograma que muestra intensa neutropenia.

Hematocrito $30.9 \%$

G.R. HB 10.5

Micrescitosis $(++)$ Macrocitosis (++) Policromatofilia $(++)$

Anisocitosis (++)

G.B. Leucocitos $10550 \times \mathrm{mm}^{3}$

Förmula: Segmentados 0.5\%;.Linfocitos $92.5 \%$ y Monocitos 7\%.

527 Neutrófilos x mm: ${ }^{3}$

Plajuetas $145.000 \times \mathrm{mm}^{3}$

Se solicitó cupremia y ceruloplasmina cuyos valores fueron 15 y $0 \mu \mathrm{g} \%$ respectivamente. Con tratamiento, valor de ceruloplasmina se normaliza 30 días después (31.3 $\mu \mathrm{g} \%$ ). Diversos hemogramas muestran, también, normalización progresiva.

Egresa a los 12 meses 9 dias con peso/edad entre -1 y -2 DS, compensada. Evidercia retraso en talla así como en el desarrollo psicomotor.

En controles ambulatorios posteriores mantiene estado nutricional y retraso psiconotor.

\section{DISCUSION}

La deficiencia nutricional de cobre es un cuadro descrito a partir de 1964 en seres humanos, especialmente en lactantes. Se conoce que hay varios antecedentes clínicos que predisponen a la deficiencia de este metal, siendo los principales peso de nacimiento bajo, lactancia materna ausente o muy abreviada, cuadros de diarrea aguda con deshidratación, alimentación en base a leche de

\section{Gräfico 4}

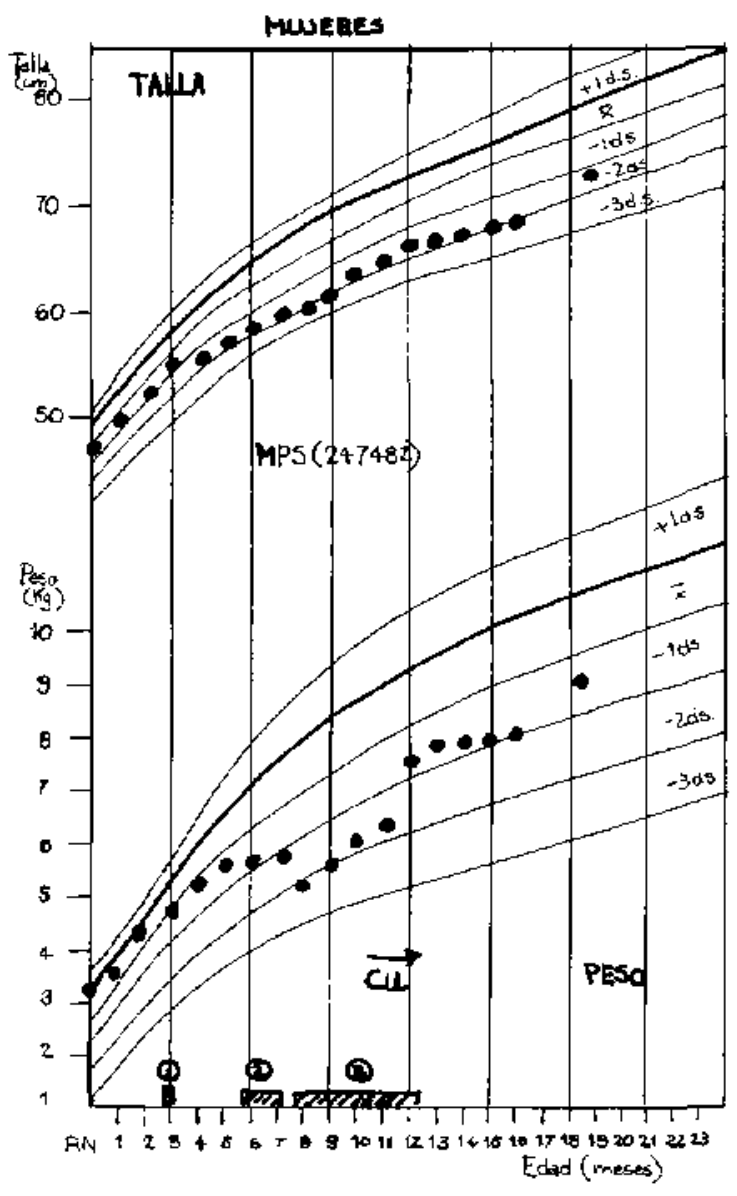

vaca en fonna prolongada y, por último, recuperación de la desnutrición. Todos estos antecedentes influyen médicamente ante disminución de las reservas prenatales de cobre, aumento de las pérdidas intestinales, aporte dietético inadecuado y aumento probable de los requerimientos en el caso de la recuperación nutricional.

En todos los casos presentados la alimentación natural no se dio o fie por un período muy breve. Se sabe que la leche materna es capaz de proporcionas los requerimientos nomales del lactante, en especial el calostro. La leche de vaca, en cambio, dada en las cantidades habituales, no cubre estos requerimientos $-80 \mu \mathrm{g} / \mathrm{Kg} / \mathrm{d}$ ía. Su contenido en este metal es varias veces inferior al de la leche humana."

También en estos niñoss se constató introducción tardia de alimentos no lácteos, especialmente cirneos, los que ofrecen un buen aporte de cobre. 2.3

El antecedente de edad gestacional de pretérmino estuvo presente en un caso y está relaciona- 
do con la menor reserva hepática, acumulación que se efectúa especialmente a partir de las 33-44 semanas de gestación. ${ }^{8}$ Lo anterior explica que el lactante de bajo peso de nacimiento corra el riesgo de deficiencia de cobre antes de los 3 meses de edad. ${ }^{1}$ En el caso anterior el diagnóstico se hizo a los 4 meses.

Otro antecedente, que en estudios previos. 5 aparece de importancia, es el de hospitalización por cuadros diareicos agudos con deshidratación en lactantes con desnutrición marásmica severa y precoz, asociación que se observó en 2 de los pacientes presentados. Todos estos pacientes tenían una desmutrición primaria severa, provocada por una situación socioeconómica muy deficiente, sobre la cual se instalaron procesos infecciosos que Ilevaron a la hospitalización. Juegan un rol importante en esta sociación, además de las pérdidas de cobre por diarrea, el aporte dietético insuficiente, por la realimentación en base a leche de vaca.

Las manifestaciones clínicas conocidas de la deficiencia de cobre están relacionadas con déficit de los sistemas enzimáticos cuprodependientes: ceruloplasmina, lisil oxidasa, superoxidismutasa, citocromo C oxidasa y otros. Son, principalmente, anemia hipocrómica, resistente a la terapia con fierro y que responde al dar cobre, neutropenia, leucopenia y alteraciones óseas, que pueden llegar a fracturas patológicas.

También podrian existir alteraciones vasculares, con ruptura o dilatación de los vasos, tanto arteriales como venosos, retardo del crecimiento, del desarrollo psicomotor, hepatoesplenomegalia, disminución de la pigmentación de la piel, episodios de apnea, hipotonía muscular y, finalmente, disminución de los valores de ceruloplasmina yfo cupremia plasmáticas. $2,3,6,7,8,9,10,11$.

En general todas estas manifestaciones requieren un tiempo relativamente prolongado y de una deficiencia acentuada para manifestarse, de modo que podemos tener toda una gama de intensidad de la sintomatología clínica.

En los casos expuestos no se constat 6 anemia, alteraciones óseas ni vasculares. En todos ellos hubo retardo acentuado del crecimiento físico y en 2 retraso psicomotor severo, que se recupera después del tratamiento sólo en un niño. Neutropenia intensa se demostró en un menor, siendo importante en la pesquisa del déficit de cobre. Finalmente, en los 4 niños los valores de cupremia fueron inferiores a $60 \mu \mathrm{g} \%$, lo que indica déficit severo. Los niveles normales en nuestro medio ${ }^{5}$ son superiores a $90 \mu \mathrm{g} \%(114 \pm 42 \mu \mathrm{g} / \mathrm{dl}$.).

Un aspecto digno de estudiar es la alta frecuencia de infecciones severas encontradas en estos pacientes, así como en otros estudiados anteriormente, y que disminuyen al suplementar con cobre. Están asociadas, al parecer, con déficit de la inmunidad celular, en especial la fagocitosis.

Parece relevante, por lo anteriomente serialado, insistir en las medidas preventivas del déficit de este mineral, que en general son comunes con las de otros oligoelementos: acciones para disminuir el bajo peso de nacimiento, promoción de la lactancia natural, evitar la alimentación láctea artificial exclusiva prolongada. Debe suplementarse con cobre los casos en que se pesquise su déficit, así como aquellos que están en riesgo de llegar a desarrollarla.

\section{RESUMEN}

En EMO (Obs 270906) el diagnóstico se efectuó a los 7 1/2 meses de vida por cupremia de $14 \mu \mathrm{g} \%$. Destacan antecedentes de DCP de III grado, que se inicia al $2 .^{\circ}$ mes de vida, escasa duración de lactancia natural, 2 hospitalizaciones previas por diarreas, la última de evolución tórpida con intolerancia a disacáridos, y ausencia de ingesta de alimentos cámeos hasta la fecha del diagnóstico. Cupremias suben a $34-91$ y $102 \mu \mathrm{g} \%$ a $\operatorname{los} 30-60$ y 90 días de tratamiento, con excelente recuperación nutricional.

En LLA (261921) no hubo alimentación natural ni ingesta de sopa hasta el diagnóstico -6 meses (cupremia $4 \mu \mathrm{g} \%$ ). También se trata de un DCP IlI grado, de iniciación precoz, con 2 hospitalizaciones previas por diartea. Excelente respuesta a] tratamiento, subiendo Cupremia a $101 \mu \mathrm{g} \%$ a los 7 $1 / 2$ meses de edad.

RMP (277543) fue pretérmino, con $2.110 \mathrm{~g}$. al nacer. No recibió pecho materno, presentando 2 hospitalizaciones, por meningitis purulenta $y$ luego infección urinaria, que lo conducen a DCP II g. descompensada. A los 4 meses 8 dias se constata cupremia de $47 \mu \mathrm{g} \%$ la que se nomaliza con tratamiento ( $118 \mu \mathrm{g} \%$ a los 90 dias).

MPS (247432) tampoco recibe pecho, desnutriéndose desde los 4 meses. Ingresa a CONIN a los 8 meses con DCP III. Previamente tuvo 4 bronconeumonías, requiriendo 2 hospitalizacio nes. A raíz de nuevo cuadro respiratorio se constató neutropenia intensa ( 55 granulocitos por $\mathrm{mm}^{3}$ ) 
a los 10 meses, investigándose déficit de cobre. Se constató ceruloplasmina $0 \mu \mathrm{\mu} \%$ y cupremia de $\mathbf{1 5}$ $\mu \mathrm{g} \%$ a los $10 \mathrm{l} / 2$ meses. Evolución posterior tarnbién tavorable.

En los casos resumidos se presentan sus características clínicas y evolución antropométrica pre y post-tratamiento con sulfato de cobre.

Se destacan las condiciones cyue pueden conducir a déficit de cobre en el lactante, revisando sintomatología clínica, prevención y tratamiento de esta patología nutricional.

\section{HEFERENCIAS}

I A/ Rashid R. :Neonatal copper deficiency". N. Fnygland. J. Med. Vol. 285 N." 15: 84 1-343, I971.
2 Bergmann $K$ and Fomons. "Copper" en Fomon "Intant Nutrition" 324-326. Salunders, 1974.

3 Casilho Durur C., Fisberg M. y Uary R.: "El cubre onmo mineral esencial para la nutrición humana" (por publicarse).

4 Cordono A., Grahon G.G.: "Copper deticiency complicating severe: sonic intestinal mala ahsonption". Pediatrics 38 . 546-

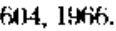

5 Fisberg M., Castilto Duran C., Egona LI y Lary R. "Factores condicionantes de hipocupremia en lautantes narásmicos". Rev, Chıl. Ped, \{en prerisa).

is Graham G. and Cordano A. 'Copper depletion and deficiency in the wainourished infant". Joluns Hopkins Med. J. 124-134, 1940.

7 Graham G.: "Human copper teficiency". N. Enkl. J. Yed. Vol, 285 N, ${ }^{\circ}$ I5: 857-858, $197 \mathrm{I}$.

A Hambidge M.: La importancia de los oliganelenentus en la nutrición infantil: Curr Med. Hes. Opin 4 Suppl, !: $48,1976$.

"O'Dell B.: "Cobre". Ein conocimientos autuales en Nutriciśn: 3/25 Incap - Alan, 1978.

19 Show d: " Frace elemtents in the Fetus and Youmk intint". Arn. J. Dis. Child. 134: 74-80, 19(4).

it Solomons N.: "On the insessment of cine and copper nutriture in ouau". Am. J. Cilis, Nutr. 32: 87 1, 1979. 